\title{
Zip property of certain ring extensions
}

\author{
André Leroy and Jerzy Matczuk * \\ Université d'Artois, Faculté Jean Perrin \\ Rue Jean Souvraz 62307 Lens, France \\ E-mail: andre.leroy@univ-artois.fr \\ Institute of Mathematics, Warsaw University \\ Banacha 2, 02-097 Warsaw, Poland \\ E-mail: jmatczuk@mimuw.edu.pl
}

\begin{abstract}
The aim of the paper is to investigate the behavior of the right zip property under some ring constructions. It includes actions of Hopf algebras, rings of quotients and subrings of matrix rings.
\end{abstract}

keywords: Zero intersection property, Hopf algebra actions, trace maps, extension of rings.

MSC: Primary: 16P20, 16S40, 16S50, Secondary: 16U20

\section{Introduction and preliminary remarks}

Throughout the paper a ring means an associative ring with unity. For any subset $S$ of a ring $R, r \cdot \operatorname{ann}_{R}(S)$ stands for the right annihilator of $S$ in $R$, i.e. r.ann $R(S)=$ $\{r \in R \mid S r=0\}$. The left annihilator is denoted by $\operatorname{l} \cdot \operatorname{ann}_{R}(S)$.

A ring $R$ is said to be right zip if, for any subset $S$ of $R$ with $\operatorname{r.ann}{ }_{R}(S)=0$, there exists a finite subset $S_{0}$ of $S$ such that $r \cdot \operatorname{ann}_{R}\left(S_{0}\right)=0$. In the above definition, one can equivalently require that $S$ is a left ideal of $R$. We will often use this characterization.

The notion of zip rings was introduced by C. Faith in [4] as a specialization of considerations of Zelmanowitz, J.A. Beachy and W.D. Blair (Cf.[13],[1]). This paper marked the beginning of the systematic studies of, and posing problems on the

*This research was supported by the Polish National Center of Science Grant No. DEC2011/03/B/ST1/04893. 
behavior of the zip property under various algebraic constructions. The subject has been studied by many authors (see for example [3], [6], [7], [14]). The aim of this paper is to investigate the behavior of the zip property under various kinds of ring extensions.

Section 2 is focused on the zip property in the presence of a Hopf algebra action. It is known that there are deep relations between various algebraic properties of an algebra $A$ and its subalgebra of constants $A^{H}$ under the action of a finite dimensional Hopf algebra $H$. The relations are especially strong when the smash product $A \# H$ is semiprime. We show that a similar situation concerns the right zip property. Namely $A^{H}$ is right zip if and only if $A$ is right $H$-zip. We also provide examples showing the limits of the obtained results. In particular, we show that zip and $H$-zip properties of $A$ are not equivalent. This situation differs from other finiteness conditions such as ACC or DCC on right ideals and their counterparts for $H$-stable right ideals. As a side effect we obtain an example of a semiprime ring $A$ acted by a finite group $G$ such that $A$ has ACC on right annihilators of $G$ - stable sets but it does not have $\mathrm{ACC}$ on right annihilators.

We will work in the much more general context of an action on a ring $A$ into its subring $R$, by a set of $(R-R)$-bimodule maps. This action is defined at the beginning of Section 2. The reason to work in such generality is twofold. On the one hand, in this way it is clear which properties are responsible for well behavior of the zip property, and on the other the general result offers a wider class of applications.

In Section 3 we investigate the behavior of the zip property with respect to localization and matrix ring construction. F. Cedó (Cf.[3]) proved that the zip property lifts neither to the polynomial ring $R[x]$ nor to the matrix $\operatorname{ring} M_{n}(R)$, for $n \geq 2$. However, in both of these cases, it lifts with the additional assumption that $R$ is commutative (Cf.[1],[3]). The authors of [7] proved similar results for the ring $U T_{n}(R)$ of all upper triangular matrices over $R$. In this section we show that, in fact, the ring $M_{n}(R)$ is right (left) zip if and only if the ring $U T_{n}(R)$.

We also investigate the zip property of some other subrings of $M_{n}(R)$. In particular, we show that the ring $R$ is right (left) zip if and only if the ring $D T_{n}(R)$ consisting of all upper triangular matrices $\left(m_{i j}\right)$ such that $m_{i i}=m_{j j}$, for $1 \leq i, j \leq n$, is such.

Concerning localization, we prove that, if $\mathcal{S}$ is a two-sided Ore set of regular elements of a ring $R$, then $R$ is right (left) zip if and only if $R \mathcal{S}^{-1}$ is such. Examples showing that such equivalence does not hold, when $\mathcal{S}$ is an Ore set only on one side, are provided.

In the following two propositions we collect, without proofs, a few basic properties of right zip rings (see [4] and [3]). Recall that a ring $R$ is left Kasch if every simple left $R$-module can be embedded into ${ }_{R} R$.

Proposition 1.1. (a) Any finite ring is right (and left) zip;

(b) A product of two rings $R \times T$ is right zip if and only if $R$ and $T$ are right zip;

(c) Any ring satisfying DCC on right annihilators is right zip;

(d) Any left noetherian ring is right zip;

(e) Any left Kasch ring is right zip. 
Proposition 1.2. Let $R$ be a subring of a right zip ring T. Then $R$ is right zip in any of the following cases:

(a) Every nonzero right ideal of $T$ intersects $R$ non-trivially, i.e. $R_{R}$ is an essential submodule of the right $R$-module $T_{R}$;

(b) T satisfies the descending chain condition on right annihilators;

(c) $T$ is free as a left $R$-module.

\section{2 zip-property under $G$-action}

Having in mind relations between the zip property of a ring and its subring of constants under the action of groups or more generally Hopf algebras, we begin this section with a general notion of an action which is suitable for our purposes.

Henceforth $R \subseteq A$ will denote an extension of rings. We say that a subset $G \subseteq \operatorname{End}\left({ }_{R} A_{R}\right)$ of $(R, R)$-bimodule endomorphisms of $A$ acts on $A$ into $R$ if, for any $\sigma \in G, \sigma(1) \in R$.

A subset $S$ of $A$ is called $G$ - stable if $\sigma(S) \subseteq S$, for any $\sigma \in G$. Notice that, due to the assumption that $\sigma(1) \in R$ for $\sigma \in G$, every one-sided ideal of $R$ is $G$-stable. Examples of such actions will be presented later in this section. Let us only remark that if $G$ is a Hopf algebra acting on an algebra $A$ and $R=A^{G}$ is the subalgebra of constants, then $G$ acts on $A$ into $R$ in the just defined manner.

A map $t \in \operatorname{End}\left({ }_{R} A_{R}\right)$ is called a non-degenerate trace if $t(A) \subseteq R$ and $0 \neq t(I) \subseteq$ $I$, for any nonzero one-sided $G$-stable ideal $I$ of $A$. Notice that conditions imposed on $t$ imply that every nonzero one- sided ideal of $A$ has non-zero intersection with $R$.

Finally, $A$ is right $G$-zip if for any $G$-stable left ideal $S$ of $A$ with $\operatorname{r.ann}_{A}(S)=0$ there exists a finite subset $S_{0} \subseteq S$ such that $\operatorname{r} \cdot \operatorname{ann}_{A}\left(S_{0}\right)=0$.

Keeping the above notation we have:

Theorem 2.1. Let $R \subseteq A$ be an extension of rings and $G \subseteq \operatorname{End}\left({ }_{R} A_{R}\right)$ acts on $A$ into $R$. Suppose that there exists a non-degenerate trace $t \in \operatorname{End}\left({ }_{R} A_{R}\right)$. Then:

(a) If $S \subseteq R$ is such that $\mathrm{r} \cdot \operatorname{ann}_{R}(S)=0$, then $\mathrm{r} \cdot \operatorname{ann}_{A}(S)=0$;

(b) $R$ is right zip if and only if $A$ is right $G$-zip.

Proof. (a) Let $S \subseteq R$ be such that $\operatorname{rann}_{R}(S)=0$ and $U=\mathrm{r} \cdot \operatorname{ann}_{A}(S)$. $U$ is a right ideal of $A$ and, for any $\sigma \in G$, we have $0=\sigma(S U)=S \sigma(U)$. This shows that $\sigma(U) \subseteq U$, i.e. $U$ is $G$-stable. Since we also have $0=t(S U)=S t(U)$, the hypothesis that $t$ is non-degenerate implies that $U=0$, as required.

(b) First let us suppose that $A$ is right $G$-zip. Let $S \subseteq R$ be a left ideal of $R$ such that $r \cdot \operatorname{ann}_{R}(S)=0$. Part (a) above shows that r. $\operatorname{ann}_{A}(S)=0$ and hence also $\operatorname{r.ann}_{A}(A S)=0$. Notice that $\sigma(A S)=\sigma(A) S \subseteq A S$, for any $\sigma \in G$. Now, the fact that $A$ is $G$-zip implies that there exists a finite subset $S^{\prime}=\left\{s_{1}, \ldots, s_{l}\right\} \subseteq A S$ such that $\mathrm{r} \cdot \operatorname{ann}_{A}\left(S^{\prime}\right)=0$. Writing elements of $S^{\prime}$ as $s_{i}=\sum_{j=1}^{l_{i}} a_{i j} s_{i j}$, for $1 \leq i \leq l$ and $a_{i j} \in A, s_{i j} \in S$, we easily conclude that $\mathrm{r} \cdot \operatorname{ann}_{A}\left(S_{0}\right)=0$, where $S_{0}=\left\{s_{i j} \mid 1 \leq i \leq\right.$ $\left.l ; 1 \leq j \leq l_{i}\right\} \subseteq S$. Hence $R$ is right zip, as required. 
Let us now suppose that $R$ is right zip and consider a $G$-stable left ideal $S$ of $A$ such that $\operatorname{r} \operatorname{ann}_{A}(S)=0$. If $a \in R$ is such that $t(S) a=0$ then $t(S a)=0$. On the other hand, for any $\sigma \in G$ we have $\sigma(S a)=\sigma(S) a \subseteq S a$. Hence, the fact that $t$ is non-degenerate implies that $S a=0$ and $a=0$ follows. In other words, r. $\operatorname{ann}_{R}(t(S))=0$. By the hypothesis, there exists a finite subset $S_{0} \subseteq t(S) \subseteq S$ such that $\mathrm{r} \cdot \operatorname{ann}_{R}\left(S_{0}\right)$. Now, the statement (a) gives the thesis.

Let $G$ be a finite group acting on a ring $A$ and $R=A^{G}=\{r \in A \mid \sigma(r)=$ $r$, for all $\sigma \in G$ \} denote the subring of constants. Then $G$ acts on $A$ into $R$. The trace of $G$ on $A$ is the function $t_{G}: A \rightarrow R$ given by $t_{G}(r)=\sum_{\sigma \in G} \sigma(r)$. Our definition of a non-degeneracy of the trace differs from the one given by S. Montgomery in the book [10]. Namely, $t_{G}$ is non-degenerate in the sense of [10] if and only if it is non-degenerate in our sense and $R=A^{G}$ is a semiprime ring. It is known (Cf. [10]) that the trace is always non-degenerate, in the sense of [10], provided $R$ is semiprime and either $R$ is $|G|$-torsion-free or $G$ is $X$-outer. Theorem 2.1 leads the following corollary.

Corollary 2.2. Let $G$ be a finite group of automorphisms of a ring $A$ such that the trace $t_{G}$ of $G$ on $A$ is non-degenerate. Then $A^{G}$ is right zip if and only if $A$ is right G-zip.

The following example shows that the assumption on the trace of $G$ is essential in Theorem 2.1 even in the case of an action of a finite group.

Example 2.3. Let $K$ be a field of characteristic different from 2. Let $R=K\left[x_{1}, x_{2}, \ldots\right]$ and $I$ be the $K$-linear vector space with basis $\left\{v_{i} \mid i \geq 1\right\}$. We define a structure of an $(R-R)$-bimodule on I by setting

$$
x_{k} v_{l}=v_{l} x_{k}=\left\{\begin{array}{l}
v_{k} \text { if } k=l \\
0 \text { else }
\end{array} .\right.
$$

Consider the commutative ring $A=R \oplus I$ with $I^{2}=0$. The map $\sigma: A \rightarrow A$ defined by $\left.\sigma\right|_{R}=i d_{R}$ and $\left.\sigma\right|_{I}=-i d_{I}$ is an automorphism of $A$ of order 2. Moreover $A^{G}=R$, where $G=\langle\sigma\rangle$.

Let $X=\left\{x_{i} \mid i \geq 1\right\}$ and $S=A X$. Then $S$ is a $G$-stable ideal of $A$. Since $S$ contains $X, \operatorname{r} \cdot \operatorname{ann}_{A}(S)=0$ and it is easy to check that any finite subset of $S$ has nonzero right annihilator in $A$. Thus $A$ is not right $G$-zip, however the ring of constants $A^{G}=R$ is a domain, so it is right zip.

Let $t_{G}$ denote the classical trace of $G$, i.e. $t_{G}=i d_{A}+\sigma$. Notice that $t_{G}(I)=0$ (but $\left.t_{G}(S)=R X\right)$.

The following example offers a ring $A$ acted by a cyclic group $G$ of order 2 such that $A$ is right zip (so it is also right $G$-zip) but the ring of constants $A^{G}$ is not right zip.

Example 2.4. Let $K$ be a field and $I$ be the ideal $\left(X_{i} X_{j} \mid 1 \leq i \leq j\right)$ of the free $K$ algebra $K\left\langle X_{1}, X_{2}, \ldots\right\rangle$. Let $R$ be the $K$-algebra $K\left\langle X_{1}, X_{2}, \ldots\right\rangle / I$ and put, for $i \geq 1$, $x_{i}=X_{i}+I$. 
Since for any nonzero element $w \in A$ there exists an index $l>0$ such that $x_{l} w \neq 0$, the set $S=\left\{x_{1}, x_{2}, \ldots\right\}$ has zero right annihilator in $R$. On the other hand, for any finite subset $S_{0}$ of $S$, there exists $k>1$ such that $S_{0} x_{k}=0$. Thus $R$ is not right zip.

Let $A=R[Y] / J$, where $J$ denotes the ideal $\left(Y^{2}, x_{1} Y, x_{2} Y, \ldots\right)$ of the polynomial algebra $A[Y]$.

Suppose now that characteristic of $K$ is different from 2. Let $\sigma$ be the automorphism of $R[Y]$ given by $\sigma \mid R=i d_{R}$ and $\sigma(Y)=-Y$. Since $\sigma(J)=J, \sigma$ induces an automorphism, also denoted by $\sigma$, of the algebra $A=R[y]$, where $y=Y+J$. Using $y^{2}=0$, one can see that $A^{<\sigma>}=R$.

Notice that $A=K \oplus M$, where $M$ denotes the ideal $\left(y, x_{1}, x_{2}, \ldots\right)$ of $A, M y=0$ and every element $c+w, 0 \neq c \in K, w \in M$ is regular in $A$. Therefore, if $S$ is a subset of $A$ such that $\mathrm{r} \cdot \operatorname{ann}_{A}(S)=0$, then $S$ is not contained in $M$ so it has to contain a regular element. Thus $A$ is an example of a right zip algebra such that the subalgebra of constants $A^{<\sigma>}=R$ is not right zip.

A classical result of J.Fisher (Cf.[5]) says that if a modular lattice $L$ is acted by a finite group $G$ of automorphisms and the sublattice $L^{G}$ of fixed elements satisfies any of a large class of chain conditions (including ACC and DCC), then $L$ satisfies the same condition. This theorem applies to the lattice of right ideals of a ring $R$ acted by a finite group $G$ and shows that $R$ has ACC (DCC) on right ideals if and only if it has the same property for $G$-stable right ideals. The following example shows that even if the $G$ action is as good as possible i.e. $A$ is semiprime and $|G|$ is invertible in $A$, then the fact that $A$ is right $G$-zip does not imply that $A$ is right zip.

Let us also recall (see 5.8[10]) that when $A$ is as described above and $A^{G}$ is right (left) noetherian, then so is $A$. In Example 2.5, $A^{G}$ is a domain so it is right zip but $A$ is not right zip. In particular, DCC on right annihilators does not lift from $A^{G}$ to A.

Example 2.5. Let $K$ be a field and define $A=K\left\langle X_{i}, Y_{j} \mid i, j \in \mathbb{N}\right\rangle / I$ where $I$ is the ideal generated by all monomials $X_{i} X_{j}$ and $Y_{i} Y_{j}$ with $1 \leq i \leq j$. Notice that the algebra $A$ is the free product of two copies of the algebra $R$ from Example 2.4.

As usual we denote $x_{i}=X_{i}+I$ and $y_{i}=Y_{i}+I$, for $i \in \mathbb{N}$. Let $\sigma$ be the $K$ automorphism of $A$ given by $\sigma\left(x_{i}\right)=y_{i}$ and $\sigma\left(y_{i}\right)=x_{i}$, for all $i \geq 1$ and $G=\langle\sigma\rangle$. Then:

(a) $\mathrm{r} \cdot \operatorname{ann}_{A}(\gamma, \sigma(\gamma))=1 . \operatorname{ann}_{A}(\gamma, \sigma(\gamma))=0$, for any nonzero $\gamma \in A$;

(b) Nonzero elements of $A^{G}$ are regular in $A$ (and hence $A^{G}$ is a domain, so it is right zip);

(c) Let $t_{G}=i d_{A}+\sigma$. Then $t_{G}(I) \neq 0$, for any nonzero one-sided ideal I of $A$. In particular, the trace $t_{G}$ is non-degenerate;

(d) A is a semiprime algebra which is right $G$-zip but it is not right zip;

(e) Neither DCC on right annihilators nor the right zip property lifts from $A^{G}$ to A. 
Proof. Let us define the degree of a word in $x_{i}$ 's and $y_{i}$ 's as the number of letters which appear in its reduced presentation. Notice that for two words $v, w$, either $\operatorname{deg} v w=\operatorname{deg} v+\operatorname{deg} w$ or $v w=0$. The degree of nonzero elements from $K$ is set to be 0 . For $0 \neq \gamma \in A$, the degree $\operatorname{deg} \gamma$ of $\gamma$ is the minimal degree of nonzero monomials appearing in $\gamma$.

(a) Let us first remark that, for $s \geq 1$ and a nonzero word $m \in A x_{s}$, we have r. $\operatorname{ann}_{A}(m)=\sum_{j \geq s} x_{j} A$. Similarly if $m \in A y_{s}$, then r.ann $\operatorname{an}_{A}(m)=\sum_{j \geq s} y_{j} A$. This implies that for any nonzero word $m, \operatorname{r} \cdot \operatorname{ann}_{A}(m, \sigma(m))=0$ as contained in $\sum_{j \geq s} x_{j} A \cap \sum_{j \geq s} y_{j} A=0$.

Let $0 \neq \gamma \in A$ and $r \in A$ be such that $\gamma r=\sigma(\gamma) r=0$. We claim that $r=0$.

If $\operatorname{deg} \gamma=0$ we can write $\gamma=k+s$ and $r=k^{\prime}+s^{\prime}$ where $0 \neq k \in K, k^{\prime} \in K$ and $s, s^{\prime}$ are elements of the ideal of $A$ generated by the set $\left\{x_{i}, y_{i} \mid i \geq 1\right\}$. From $\gamma r=0$ we immediately get that $k^{\prime}=0$. Let $m^{\prime}$ denote the sum of monomials of $s^{\prime}$ of minimal degree. Since either $s m^{\prime}=0$ or all monomials from $s s^{\prime}$ have degree greater than $\operatorname{deg} s^{\prime}$, the equality $\gamma r=k s^{\prime}+s s^{\prime}=0$ implies that $k s^{\prime}=0$. This leads to $s^{\prime}=0$ and hence $r=0$. Notice that we actually proved that any element with nonzero independent term is right regular in $A$. Using a similar argument on the left, one can check that such an element is also left regular.

Suppose $\operatorname{deg} \gamma=l \geq 1$. Let us write $\gamma_{l}=\gamma_{l 1}+\cdots+\gamma_{l s}$ for the sum of all monomials from $\gamma$ of degree $l$ and $r_{m}$ for the sum of all monomials of $r$ of degree $\operatorname{deg} r$. Since, by hypothesis, we have $\gamma r=\sigma(\gamma) r=0$ we also have $\gamma_{l} r_{m}=\sigma\left(\gamma_{l}\right) r_{m}=0$. Notice also that the sum $\sum_{i=1}^{s} \gamma_{l i} A$ is direct, since all monomials $\gamma_{l i}$ 's are distinct and have the same degree. This, in particular, implies that $\gamma_{l 1} r_{m}=\sigma\left(\gamma_{l 1}\right) r_{m}=0$. However $\gamma_{l 1}$ is a monomial and we have seen above that this implies that $r_{m}=0$ and hence $r$ must be equal to zero. This shows that $\mathrm{r}_{\operatorname{ann}}(\gamma, \sigma(\gamma))$.

Now if $r \in 1 . \operatorname{ann}_{A}(\gamma, \sigma(\gamma))$ we have $r \gamma=r \sigma(\gamma)=0$ and hence also $r \gamma=\sigma(r) \gamma=0$ i.e. $\gamma \in \mathrm{r}$. $\operatorname{ann}_{A}(r, \sigma(r))$ and what we just proved implies that if $\gamma \neq 0$ then $r=0$

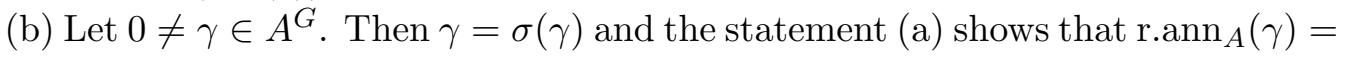
l.ann ${ }_{A}(\gamma)=0$.

(c) Notice that, if $t_{G}(m)=m+\sigma(m)=0$, for an $m \in A$, then $\sigma(m)=-m$. Thus, the statement (a) yields that if $0 \neq m \in A$ satisfies $t_{G}(m)=0$, then $m$ is (left and right) regular in $A$.

Let $I$ be a left ideal of $A$ such that $t_{G}(I)=0$. Assume that $0 \neq m \in I$, then, as we just noticed, $m$ is regular. Since $x_{1} m \in I$ and $x_{1}\left(x_{1} m\right)=0$, we must have $x_{1} m=0$. This contradiction shows that $t(I) \neq 0$, for any nonzero left ideal $I$ of $A$.

A similar argument applied to right ideals shows that $t(I) \neq 0$, for any nonzero right ideal $I$ of $A$. This completes the proof of (c).

(d) Statements (b) and (c) imply that every nonzero $G$-stable one-sided ideal of $A$ contains a regular element. In particular, if the prime radical $B(A)$ of $A$ would be non-zero, it would contain a regular element. Thus $B(A)=0$, i.e. $A$ is semiprime.

Obviously $A$ has to be right $G$-zip, since every $G$-stable left ideal of $A$ contains a regular element.

Taking $S=\left\{x_{i} \mid i \geq 1\right\}$ and applying the same arguments as in Example 2.4, one can check that $A$ is not right zip.

(e) By (b), the subalgebra of constants $A^{G}$ is a domain, thus has DCC on right 
annihilators. The fact that every ring with DCC on right annihilators is right zip and the statement (d) complete the proof of (e).

In the next proposition we show that Theorem 2.1 can be applied to infinite groups of automorphisms (so there is no natural trace map in that case).

For a ring $R$ and natural number $n, M_{n}(R)$ will denote the $n \times n$ matrix ring over $R$ and $\left\{e_{i j} \mid 1 \leq i, j \leq n\right\}$ will stand for the set of matrix units of $M_{n}(R)$. We will consider $R$ as embedded in $M_{n}(R)$ via diagonal matrices. Let us define $t: M_{n}(R) \rightarrow R$ by setting $t\left(\left(m_{i j}\right)\right)=\operatorname{diag}\left(m_{11}, m_{11}, \ldots, m_{11}\right)$. Notice that $t$ is a homomorphism of $(R-R)$-bimodules.

Proposition 2.6. Let $G$ be the set of (or the group generated by) all inner automorphisms of $M_{n}(R)$ adjoint to all elements of the form $1+e_{k l}$, where $1 \leq k, l \leq n$ and $k \neq l$. Then $R$ is right zip if and only if $M_{n}(R)$ is right $G$-zip.

Proof. First let us observe that any $G$-stable left (resp. right) ideal of $M_{n}(R)$ is of the form $M_{n}(I)$, where $I$ is a left (resp. right) ideal of $R$. To this end, let $L$ be a $G$-stable left ideal of $M_{n}(R)$. For any $1 \leq k, l \leq n$ with $k \neq l$, we have $L=$ $\left(1+e_{k l}\right) L\left(1-e_{k l}\right)=L\left(1-e_{k l}\right)$. Hence, for any $M \in L, M e_{k l}=M-M\left(1-e_{k l}\right) \in L$. This gives also $M e_{k k}=\left(M e_{k l}\right) e_{l k} \in L$. Therefore, for $M=\left(m_{i j}\right) \in L$, we get $m_{i j} e_{11}=e_{1 i} M e_{j 1} \in L$. The above easily yields that $L=M_{n}(I)$ where $I$ is the left ideal $\left\{m_{11} \in R \mid M=\left(m_{i j}\right) \in L\right\}$ of $R$. Similar arguments prove the statement for right $G$-stable ideals of $M_{n}(R)$.

Now it is clear that for any nonzero $G$-stable one-sided ideal $L$ of $M_{n}(R)$ we have $0 \neq I=t(L) \subseteq L$, where $I$ is the one-sided ideal of $R$ such that $L=M_{n}(I)$. Thus $t$ is a non-degenerate trace and Theorem 2.1 gives the thesis.

Let us notice that in the case $R$ is an algebra over a field of nonzero characteristic $p$ dividing $n$ then the trace from the above proposition is still non-degenerate but this would not be the case of the usual trace map.

We have seen in Example 2.5 that the right zip and the right $G$-zip properties are not equivalent when $G$ is a finite group acting by automorphisms. Another such example, in the case of nonzero characteristic, can be obtained as follows: F. Cedó [3], using a result of G. Bergman [2], proved that there exists a $K$-algebra $D$ which is a domain such that $M_{n}(D)$ is not right zip. Let $K$ be a field of nonzero characteristic and consider $R=M_{n}(D)$ the $K$-algebra constructed by F. Cedó. Notice that the group $G$ from Proposition 2.6 is then finite and this proposition implies that $R$ is right $G$-zip. We believe that it would be worth not only to know that such a domain $D$ exists but also to have a concrete example. Let us notice that if $D$ is such, then $D$ can not be embedded into division ring, as otherwise $M_{n}(D)$ would be a subring of simple artinian ring thus it would be left and right zip.

Theorem 2.1 can be also applied in more general setting of actions of finite dimensional Hopf algebras. Let $H$ be a finite dimensional Hopf algebra over a field $K$ and $A$ be a left $H$-module $K$-algebra. Recall that this means that $A$ has a structure of a left $H$-module such that the action of $H$ on $A$ behaves well with respect to the coproduct and the co-unity of $H$. For more information on this topic the reader may 
consult [11]. The subalgebra $A^{H}$ of constants consists of all elements $a \in A$ such that $h \cdot a=\epsilon(h) a$, for any $h \in H$, where $\epsilon$ denotes the counit of $H$.

It is known that $H$ admits a nonzero left integral $t$, i.e. an element $0 \neq t \in H$ such that $h t=\epsilon(h) t$, for any $h \in H$ and the map $\hat{t}: A \rightarrow A$ defined by $\hat{t}(a)=t \cdot a$ is a homomorphism of $\left(A^{H}-A^{H}\right)$-bimodules with values in $A^{H}$.

We close this section with the following special case of Theorem 2.1.

Corollary 2.7. Let $H$ be a finite dimensional Hopf $K$-algebra and $A$ a left $H$-module algebra. Suppose that $t \in H$ is a left integral such that $\hat{t}(I) \neq 0$, for any nonzero $H$-stable one-sided ideal $I$ of $A$. Then $A$ is right $H$-zip if and only if $A^{H}$ is right zip.

Let us remark that, due to Lemma 4.4.6. of [11], the assumptions of the above corollary are fulfilled when the smash product $A \# H$ is a semiprime algebra.

Moreover, it is known that when $A$ is semiprime then $A \# H$ is semiprime in any of the following cases:

(i) $H=K G$ is a group algebra and $|G|^{-1} \in K$ (Cf.[11]);

(ii) $H=(K G)^{*}$ is the dual of a group algebra and $|G|^{-1} \in K$ (Cf.[11]);

(iii) $H$ is any semisimple Hopf algebra and $A$ satisfies a polynomial identity (Cf.[9]).

It is an open problem, posed by M.Cohen, whether $A \# H$ is semiprime provided $A$ is semiprime and $H$ is semisimple.

In the case $H=(K G)^{*}$, Corollary 2.7 can be read as follows:

Corollary 2.8. Let $G$ be a finite group with the neutral element e and $A=\bigoplus_{g \in G} A_{g}$ be a G-graded $K$-algebra. Suppose that every nonzero one-sided homogenous ideal has nonzero intersection with $A_{e}$. Then $A_{e}$ is right zip if and only if any homogenous left ideal of $A$ having zero right annihilator contains a finite subset $S$ such that r.ann ${ }_{A}(S)=0$.

\section{Localization and matrix rings.}

In this section we investigate the behavior of the zip property for other kinds of ring extensions. We begin with the following technical observation, which appears to be useful in applications.

Proposition 3.1. Let $R \subseteq A$ be a ring extension such that:

(i) $R$ is essential as right $R$-submodule of $A$;

(ii) r.ann $R(I \cap R)=0$, for any left ideal $I$ of $A$ such that $\mathrm{r} \operatorname{ann}_{A}(I)=0$.

Then $R$ is right zip if and only if $A$ is right zip.

Proof. Proposition 1.2 and the assumption ( $i$ ) imply that $R$ is right zip provided $A$ is such.

Suppose $R$ is right zip. Let $I$ be a left ideal of $A$ with r.ann ${ }_{A}(I)=0$. Then, by (ii), we can pick a finite subset $I_{0}$ of $I \cap R$ such that r. $\operatorname{ann}_{R}\left(I_{0}\right)=0$. Observe that r.ann $A_{A}\left(I_{0}\right)$ has to be equal to zero, as every nonzero right ideal of $A$ has nonzero intersection with $R$. This shows that $A$ is right zip in this case. 
Notice that the above proposition applies when $R \subseteq A$ is a ring extension such that, for any left ideal $I$ and right ideal $J$ of $A$, one has $I=A(I \cap R)$ and $J=(J \cap R) A$. Therefore, as a corollary, we obtain the following theorem.

Theorem 3.2. (1) Let $\mathcal{S}$ be a two-sided Ore set of regular elements of a ring $R$. Then $R$ is right (left) zip if and only if the ring of quotients $R \mathcal{S}^{-1}$ is right (left) zip;

(2) The ring $M_{n}(R)$ is right (left) zip if and only if $U T_{n}(R)$ is right (left) zip.

Proof. (1) It is known and easy to check that if $\mathcal{S}$ is a right Ore set of regular elements of a ring $R$ and $J$ is a right ideal of $R \mathcal{S}^{-1}$, then $J=(J \cap R) R \mathcal{S}^{-1}$. Moreover, when $\mathcal{S}$ is a two-sided Ore set, then the rings $R \mathcal{S}^{-1}$ and $\mathcal{S}^{-1} R$ are isomorphic. Therefore, in this case we also have $I=R \mathcal{S}^{-1}(I \cap R)$, for any left ideal $I$ of $Q$. Now, the thesis is a consequence of Proposition 3.1.

(2) Let $A=M_{n}(R)$ and $T=U T_{n}(R)$. For any right ideal $J$ of $A$, we have $\sum_{k=1}^{n} J e_{k n} \subseteq J \cap T$ and $J \subseteq\left(\sum_{k=1}^{n} J e_{k n}\right) A \subseteq(J \cap T) A \subseteq J$.

Similarly, when $I$ is a left ideal of $A$, then $I \subseteq A\left(\sum_{j=1}^{n} e_{1 j} I\right) \subseteq A(T \cap I) \subseteq I$. Now the statement (2) is a consequence of Proposition 3.1.

F. Cedó [3] investigated the relation between zip property of a ring $R$ and the matrix ring $M_{n}(R)$. Parallel results, for the ring of upper triangular matrices $U T_{n}(R)$, were obtained by C.Y. Hong, N.K. Kim, T.K. Kwak, Y. Lee in [7]. The above theorem says that the results are, in fact, equivalent.

In examples below we will be using skew polynomial rings and in this context it is more natural to consider left zip property. If someone wants to obtain similar examples on the right hand side, it is enough to consider skew polynomial rings with coefficients written on the right of the indeterminate.

If $\alpha$ is an automorphism of a ring $R$ and $W$ denotes either the skew polynomial ring $R[x ; \alpha]$ or the skew Laurent polynomial ring $R\left[x, x^{-1} ; \alpha\right]$, then $W$ is free as a left and right $R$-module. Thus, Proposition 1.2 and its left hand version yield that if $W$ is zip on one side, then the base ring $R$ is also zip on the same side.

Let $\sigma$ be an endomorphism of $R$. Then the skew polynomial ring $R[x ; \sigma]$ (with coefficients written on the left of the indeterminate) is free as a left $R$-module. Thus, when $R$ is not right zip, then also $R[x ; \sigma]$ is not right zip. If, in addition, $\sigma$ is injective but not onto, then the right $R$ - submodule $M$ of $R[x ; \sigma]$ spanned by all powers of $x$ is free but $M \neq R[x ; \sigma]$. In the following example we show that in such a situation it may happen that $R[x ; \sigma]$ is left zip (but it is not right zip), but nevertheless $R$ is not left zip. This example will be used later on for showing that the zip property does not have to be preserved under one-sided localization.

Example 3.3. Let $K$ be a field and $R$ a commutative $K$-algebra with basis $\{1\} \cup B$, where $B=\left\{e_{i} \mid i=1,2, \ldots\right\}$ and $e_{i}$ 's are orthogonal idempotents (i.e. $R$ is isomorphic to subalgebra with unity of $\prod_{i=1}^{\infty} K$ generated by $\left.\bigoplus_{i=1}^{\infty} K\right)$. Then $l_{\cdot a_{n}}(B)=$

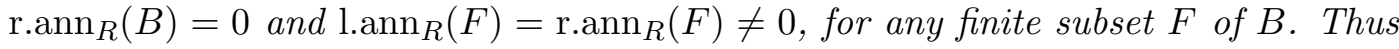
$R$ is neither left nor right zip.

Let $T=R[x ; \sigma]$, where $\sigma$ is the $K$-endomorphism of $R$ defined by $\sigma\left(e_{i}\right)=e_{i+1}$, for any $i \geq 1$. Notice that each element $w(x)$ of $T$ can be written in a form $w(x)=$ 
$k(x)+b(x)$ where $k(x) \in K[x], b(x) \in I T$ and $I$ is the ideal of $R$ generated by the set $\left\{e_{i}\right\}_{i \geq 1}$. Then $e_{1} t I T=0$ and $\operatorname{l.ann}_{T}(w(x))=0$ if and only if $k(x) \neq 0$. This implies that $T$ is left zip. Notice also that $T$ is not right zip, as otherwise, by Proposition 1.2(c), $R$ would be right zip.

When $\mathcal{S}$ is a left Ore set of regular elements of $R$, then $R$ is essential as a submodule of a left $R$-module $\mathcal{S}^{-1} R$. Thus, if $\mathcal{S}^{-1} R$ is left zip, then so is $R$. The following example shows that the reverse implication does not have to hold, when $\mathcal{S}$ is not a two- sided Ore set of $R$ (compare with Theorem 3.2).

Example 3.4. Let $T=R[x ; \sigma]$ be the ring defined in Example 3.3. It is known that $\mathcal{S}=\left\{x^{i}\right\}_{i \geq 1}$ is a left Ore set in $T$ and $\mathcal{S}^{-1} T$ is isomorphic to the skew Laurent polynomial ring $\hat{R}\left[x, x^{-1} ; \sigma\right]$ (Cf.[8]), where $\hat{R}$ is the $K$-algebra with basis $\{1\} \cup\left\{e_{i} \mid\right.$ $i \in \mathbb{Z}\}$ and $\sigma$ is the $K$-automorphism of $\hat{R}$ given by $\sigma\left(e_{i}\right)=e_{i+1}$, for all $i \in \mathbb{Z}$.

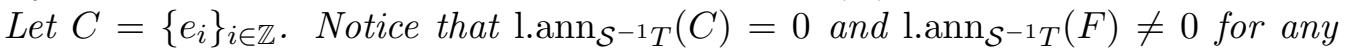
finite subset $F$ of $C$. This implies that $\mathcal{S}^{-1} T$ is not left zip. However, we have seen in Example 3.3, that $T$ is left zip.

Let $D T_{n}(R)$ denote the subring of $U T_{n}(R)$ consisting of all matrices $\left(a_{i j}\right) \in$ $U T_{n}(A)$ such that $a_{i i}=a_{j j}$, for all $1 \leq i, j \leq n$. In Theorem 5 of [7] the authors gave a relatively long, computational proof of the fact the ring $R$ is right zip if and only if the ring $D T_{n}(R)$ is such. In another theorem they proved also that $R$ is right zip if and only if the factor ring $R[x] /\left(x^{n}\right)$ is right zip. Both extensions of $R$ are examples of the situation of an over-ring $T$ of $R$ such that $T=R \oplus I$ for a certain two-sided nilpotent ideal $I$ of $T$. In general one can not suspect that the zip property of $R$ and that of $T=R \oplus I$ are related in this situation. Notice that Example 2.3 offers a commutative domain $R$ such that $T=R \oplus I$ is not right zip and $I^{2}=0$. The following example shows that the right zip property does not pass from $T=R \oplus I$ to $R$. In this example also $I^{2}=0$.

Example 3.5. Let $K$ be a field and $R=K\left\langle x_{1}, x_{2} \ldots \mid x_{i} x_{j}=0,1 \leq i \leq j\right\rangle$ be the $K$-algebra defined in Example 2.4. We have seen that $R$ is not right zip.

Let $I$ be a one dimensional $K$ vector space with the basis $\left\{x_{0}\right\}$. Then $I$ has a structure of an $(R-R)$-bimodule given by $x_{i} x_{0}=x_{0} x_{i}=0$. Let $T=R \oplus I$, with

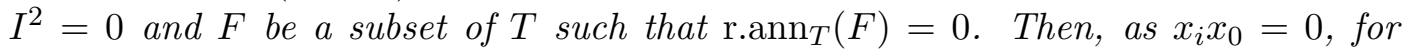
any $i \geq 1, F$ has to contain an element of the form $k+w$, where $0 \neq k \in K$ and $w \in \sum_{i=0}^{\infty} x_{i} T$. Then $\mathrm{r} . \operatorname{ann}_{T}(k+w)=0$, i.e. $T$ is right zip.

In the remaining part of the paper we will concentrate on the description of the right zip property of such $T=R \oplus I$ under some additional assumptions. The above examples show that the nilpotency of the ideal $I$ is not enough to guarantee relations between zip properties of $R$ and $T$. Therefore, in order to get positive results, we will add some extra assumptions on annihilators (Cf. Theorems 3.10 and 3.13). With this perspective let us mention, without proof, the following lemma in which we analyze different ways of expressing the condition that we will meet in these theorems.

Lemma 3.6. Let $R \subseteq T=R \oplus I$ be a ring extension where $I$ is an ideal of $T$ such that $I^{n}=0$ for some $n \geq 2$. Then the following are equivalent: 
(i) $\operatorname{l.ann}_{R}\left(I^{n-1}\right)=0$, i.e. $I^{n-1}$ is faithful as a left $R$-module;

(ii) $\operatorname{l.ann}_{T}\left(I^{n-1}\right)=I$;

(iii) For every $1 \leq k \leq n-1, I^{k} / I^{k+1}$ is faithful as a left $R$-module;

(iv) For every $1 \leq k \leq n-1$, $1 \cdot \operatorname{ann}_{T}\left(I^{k} / I^{k+1}\right)=I$.

We begin our investigations on the behavior of the zip property of extensions $R \oplus I$ with the following general observation.

Proposition 3.7. Let $R \subseteq W$ be a ring extension. Suppose that $W$ can be embedded, as a left $R$-module, in a free left $R$-module. If $W$ is right zip, then so is $R$.

Proof. Let $F$ be a free left $R$ module such that ${ }_{R} W \subseteq_{R} F$ and $S$ be a subset of $R$ such that $r \cdot \operatorname{ann}_{R}(S)=0$. Then, the freeness of ${ }_{R} F$ implies that $r \cdot \operatorname{ann}_{F}(S)=0$. Hence also r.ann $\operatorname{an}_{W}(S)=0$, as $W$ is a submodule of ${ }_{R} F$. Thus, if $W$ is right zip, we can find a finite subset $S_{0}$ of $S$ with r. $\operatorname{ann}_{W}\left(S_{0}\right)=0$. This gives the thesis.

As a direct application of the above, let us record the following corollary.

Corollary 3.8. Let $W$ be a subring of $M_{n}(R)$ containing $R$ (as diagonal matrices). If $W$ is right zip, then so is $R$.

Lemma 3.9. Let $n \geq 2$ and $T=R \oplus I$, where $I$ is an ideal of $T$ such that $I^{n}=0$ and l. $\operatorname{ann}_{R}\left(I^{n-1}\right)=0$. If $R$ is right zip then, for any subset $S$ of $T$ such that $\mathrm{r} \cdot \operatorname{ann}_{T}(S)=$ 0 , there exist finite subsets $\bar{S}_{0}=\left\{r_{1}, \ldots, r_{m}\right\} \subseteq R$ and $S_{0}=\left\{r_{1}+w_{1}, \ldots, r_{m}+w_{m} \mid\right.$

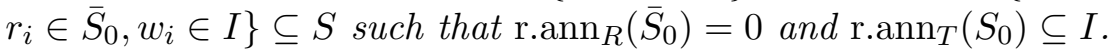

Proof. Suppose $R$ is right zip. Let $S$ be a subset of $T$ such that $\operatorname{rann}(S)=0$. Then there are an index set $\mathcal{A}$, elements $r_{i} \in R, w_{i} \in I$, for $i \in \mathcal{A}$, such that $S=\left\{r_{i}+w_{i} \mid\right.$ $i \in \mathcal{A}\}$. Let us set $\bar{S}=\left\{r_{i} \mid i \in \mathcal{A}\right\}$ and $J=\operatorname{r.ann}_{R}(\bar{S})$. Since $I$ is an ideal of $T$ and $I^{n}=0$, we have $I J I^{n-1}=0$. Hence $S J I^{n-1}=\bar{S} J I^{n-1}=0$. This shows that $J I^{n-1} \subseteq \mathrm{r} \cdot \operatorname{ann}_{T}(S)=0$. Consequently $J=0$ as, by assumption, $\operatorname{lann}_{R}\left(I^{n-1}\right)=0$. Now, since $R$ is right zip, there exists a finite subset $\bar{S}_{0}=\left\{r_{1}, \ldots r_{m}\right\} \subseteq \bar{S}$ such that r. $\operatorname{ann}_{R}\left(\bar{S}_{0}\right)=0$. Let us define $S_{0}=\left\{r_{1}+w_{1}, \ldots, r_{m}+w_{m} \mid r_{i} \in \bar{S}_{0}, w_{i} \in I\right\} \subseteq S$ and let $r+w \in \operatorname{r.ann} T\left(S_{0}\right)$, where $r \in R$ and $w \in I$. The equality $\left(r_{i}+w_{i}\right)(r+w)=0$ implies that $r_{i} r=0$, for any $1 \leq i \leq m$. This means that $r \in \mathrm{r} \cdot \operatorname{ann}_{R}\left(S_{0}\right)=0$ and shows that $\operatorname{r} \cdot \operatorname{ann}_{T}\left(S_{0}\right) \subseteq I$ as required.

Theorem 3.10. Let $n \geq 2$ and $T=R \oplus I$, where $I$ is an ideal of $T$ such that $I^{n}=0$

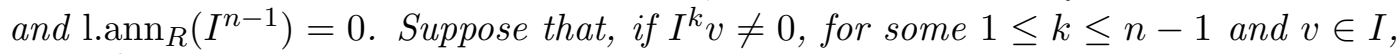
then $I^{k} S v \neq 0$, for any subset $S$ of $R$ with $\mathrm{r} \cdot \operatorname{ann}_{R}(S)=0$. If $R$ is a right zip, then so is $T$.

If additionally, for any subset $S$ of $R \mathrm{r} \cdot \operatorname{ann}_{I}(S)=0$ provided $\mathrm{r} \cdot \operatorname{ann}_{R}(S)=0$, then $R$ is right zip if and only if $T$ is right zip.

Proof. Suppose that $R$ is right zip and $S \subseteq T$ is such that $\mathrm{r} \cdot \operatorname{ann}_{T}(S)=0$. Then, making use of Lemma 3.9 we can pick finite subsets $\bar{S}_{0}=\left\{r_{1}, \ldots, r_{m}\right\} \subseteq R$ and $S_{0}=\left\{r_{1}+w_{1}, \ldots, r_{m}+w_{m} \mid r_{i} \in \bar{S}_{0}, w_{i} \in I\right\} \subseteq S$ such that $\operatorname{rann}_{T}\left(S_{0}\right) \subseteq I$ and r. $\operatorname{ann}_{R}\left(\bar{S}_{0}\right)=0$. 
Assume $0 \neq v \in \operatorname{rann}_{T}\left(S_{0}\right) \subseteq I$. Since $I$ is nilpotent and $v \neq 0$, there exists $k \geq 0$ such that $I^{k} v \neq 0$ and $I^{k+1} v=0$. Then $0=I^{k} S_{0} v$ implies $I^{k} \bar{S}_{0} v=0$ as $I^{k+1} v=0$. However, the hypotheses show that $I^{k} \bar{S}_{0} v \neq 0$. This contradicts the assumption that $v \neq 0$ and yields that $\operatorname{rann}_{T}\left(S_{0}\right)=0$. This shows that $T$ is right zip in this case.

Suppose now that $T$ is right zip and $\operatorname{r} \cdot \operatorname{ann}_{I}(S)=0$, for any subset $S$ of $R$ with r. $\operatorname{ann}_{R}(S)=0$. Let $S$ be a subset of $R$ such that $\operatorname{rann}_{R}(S)=0$ and $S(r+w)=0$, for some $r \in R$ and $w \in I$. Then, as $S r \subseteq R, S w \subseteq I, S r=S w=0$. This shows

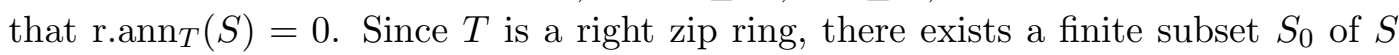
such that $\mathrm{r} \cdot \operatorname{ann}_{T}\left(S_{0}\right)=0$. Then clearly r. $\operatorname{ann}_{R}\left(S_{0}\right)=0$, i.e. $R$ is right zip.

Let us remark that the additional assumption in the above theorem holds when $I$ is isomorphic as a left $R$-module to a submodule of a free left $R$-module.

For $n=2$ the above theorem gives the following:

Corollary 3.11. Let $T=R \oplus I$, where $I^{2}=0$. Then:

(i) If $R$ is right zip and $1 \cdot \operatorname{ann}_{R}(I)=0$, then $T$ is right zip.

(ii) If $I$ is a free left $R$-module, then $R$ is right zip if and only if $T$ is right zip.

With the help of Theorem 3.10 it is easy to construct extensions of commutative domains which are right but not left zip.

Proposition 3.12. Let $R$ be a commutative domain with an $(R, R)$-bimodule $I$ such that: ${ }_{R} I$ is torsion free (i.e. if $r w=0$ and $0 \neq r \in R$, then $w=0$ ). Then the ring $T=R \oplus I$, where $I^{2}=0$, is right zip.

If additionally, there exists a subset $S$ of $R$ such that $1 \cdot \operatorname{ann}_{I}(S)=0$ and $l \cdot \operatorname{ann}_{I}\left(S_{0}\right) \neq$ 0 , for any finite subset $S_{0}$ of $S$, then $T$ is not left zip.

Proof. By Corollary 3.11, $T$ is a right zip ring.

Let $S \subseteq R \subseteq T$ be a subset satisfying assumptions. Let $a \in R$ and $w \in I$ be such that $0=(a+w) S$. Then $a=0$ and $w=0$, as $a S \subseteq R, w S \subseteq I$. However, by assumption, every finite subset $S_{0}$ of $S$ has nonzero left annihilator in $T$. Thus $T$ is not left zip.

Let $K$ be a field, $R=K\left[x_{k} \mid k \geq 0\right]$ and $I$ be the $K$-linear space with basis $\left\{v_{i j} \mid i, j \geq 0\right\}$. Then $I$ has a structure of $(K[x], K[x])$-bimodule given by

$$
x_{l} v_{i j}=v_{l+i, j}, \text { for any } l \geq 0 \quad \text { and } \quad v_{i j} x_{k}=\left\{\begin{array}{ll}
0 & \text { if } k \leq j \\
v_{i j} & \text { if } k>j
\end{array} .\right.
$$

Notice that $R, I$ and $S=\left\{x_{k} \mid k \geq 0\right\}$ satisfy the assumptions of the above proposition. Thus the ring $R \oplus I$, with $I^{2}=0$, is right but not left zip.

The following theorem nicely fits to subrings of matrix rings.

Theorem 3.13. Let $T=R \oplus I$. Suppose that $T$, as a left $R$ module, can be embedded into a free left $R$-module, $l_{\cdot \operatorname{ann}_{T}}\left(I^{n-1}\right)=I$ and $l_{\cdot} \cdot \operatorname{ann}_{T}(I)=I^{n-1}$, for some $n \geq 2$. Then $R$ is right zip if and only if $T$ is right zip. 
Proof. Suppose that $R$ is right zip. Let $S$ be a subset of $T$ such that $\operatorname{r.ann}_{T}(S)=0$. Then, making use of Lemma 3.9 we can pick finite subsets $\bar{S}_{0}=\left\{r_{1}, \ldots, r_{m}\right\} \subseteq R$ and $S_{0}=\left\{r_{1}+w_{1}, \ldots, r_{m}+w_{m} \mid r_{i} \in \bar{S}_{0}, w_{i} \in I\right\} \subseteq S$ such that $\operatorname{rann}_{T}\left(S_{0}\right) \subseteq I$ and $\operatorname{r.ann}_{R}\left(\bar{S}_{0}\right)=0$.

Let $0 \neq v \in I$ be such that $S_{0} v=0 . I$ is nilpotent, thus, multiplying $v$ on the right by suitable element from $I$ we may assume $v I=0$. Hence, by assumption $v \in I^{n-1}$ and $I v=0$. Therefore $\bar{S}_{0} v=0$. Let ${ }_{R} M$ be a free $R$-module such that ${ }_{R} T \subseteq_{R} M$. Working in $M$ it is clear, as $\operatorname{rann}_{R}\left(\bar{S}_{0}\right)=0$, that $v=0$. This contradiction yields

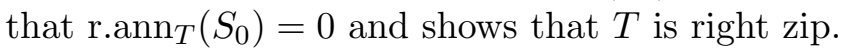

The reverse implication is given by Proposition 3.7.

Applying the above theorem to $D T_{n}(R)=R \oplus I$, where $I$ is the ideal of all strictly upper triangular matrices of $D T_{n}(R)$ and to $R[x] /\left(x^{n}\right)=R \oplus\left(x+\left(x^{n}\right)\right)$, we directly get the following corollary.

Corollary 3.14. (a) (Theorem 5 [7]) $D T_{n}(R)$ is right zip if and only if $R$ is right zip;

(b) (Proposition $9[7]$ ) $R[x] /\left(x^{n}\right)$ is right zip if and only if $R$ is right zip.

Acknowledgments We would like to thank the referee for his/her useful comments.

Part of this work was done while the first author visited Warsaw University. He would like to thank members of this institution for their warm hospitality.

\section{References}

[1] J.A. Beachy, W.D. Blair, Rings whose faithful left ideals are cofaithful, Pacific J. Math. 58 (1975) 1-13.

[2] G.M. Bergman, Modules over coproducts of rings, Trans. A.M.S. 200 (1974), $1-32$.

[3] F. Cedó, Zip rings and Mal'cev domains, Comm. Algebra 19(7) (1991), 19831991.

[4] C. Faith, Rings with zero intersection property on annihilators: zip rings, Publicacions Matemàtiques, 33 (1989), 329-338.

[5] Joe W. Fisher, Chain conditions for modular lattices with finite group actions.

[6] M. Habibi, A. Moussavi, Annihilator properties of skew monoid rings, Comm. Algebra 42 (2014), 842-852.

[7] C.Y. Hong, N.K. Kim, T.K. Kwak, Y. Lee, Extensions of zip rings, J. Pure Appl. Algebra, 195 (2005), 231-242;

[8] D. A. Jordan, Bijective extensions of injective rings endomorphisms. J. London Math. Soc. 25 (1982), 435-448.

[9] V. Linchenko, S. Montgomery, Semiprime smash products and $H$-stable prime radicals for PI- algebras, Proc. Amer. Math. Soc. 135 (2007), 3091-3098. 
[10] S. Montgomery, Fixed rings of finite automorphism groups of associative rings, Lecture Notes in Math., Springer-Verlag, 1980.

[11] S. Montgomery, Hopf Algebras and Their Actions on rings, CBMS Regional Conference Series in Mathematics vol.82, AMS, 1993.

[12] Pace P. Nielsen, Semi-commutativity and the McCoy condition, J. Algebra 298 (2006), 134-141.

[13] J. Zelmanowitz, The finite intersection property on annihilator right ideals, Proc. Amer. Math. Soc. 57 (1976), 213-216.

[14] M. Ziembowski, A note on zip rings, Acta Math. Hung. 141 (2013), 127-131. 